\section{Activities of Life and the Second Law of Thermodynamics}

I AM very glad to have elicited Prof. F. G. Donnan's critical views (NATURE, Jan. 20, p. 99) on my suggestion as to life and thermodynamics, but confess I remain unconvinced by his arguments.

Prof. Donnan challenges my neglect of the body metabolism or fuel oxidation which, as he says, necessarily accompanies the arrangement or disarrangement of material objects by human activities, considering that such chemical changes may produce an increase of entropy sufficient to offset any decrease produced by human intelligence. No doubt it may, but I cannot see that these two effects are "functionally inter-related" or in any way suitable subjects for comparison. Given perfectly level and frictionless railways, a man may move millions of tons of matter, and thereby decrease the entropy of the world enormously, without incurring any corresponding increase of entropy through the combustion of food or fuel. Any increase of entropy which occurs in practice is a mere side-issue, an accident resulting from the impossibility of realising ideal conditions, and so should not enter into the theoretical discussion at all.

A further increase of entropy might of course occur if the mental effort of arranging objects caused an increase in bodily metabolism. I believe orthodox physiology teaches that any such effect is inappreciable, but it is in any case obvious that it cannot be relied on to offset the decrease of entropy resulting from intelligent arrangement. We cannot, for example, suppose that the man who steers the Mauretania consumes food-energy at a rate comparable with 100,000 h.p. more than normal, merely because he is guiding a ship of that horsepower.

Prof. Donnan's parallel from crystal growth seems to me to fail through identifying "increase of organisation" with "decrease of entropy". The two are equivalent so long as potential energy is unimportant, but when this becomes preponderating, as in a crystal, maximum entropy may well demand regular packing, and so maximum, not minimum, organisation.

\section{J. H. JEANS.}

\section{Crystal Structure of Lanthanum, Cerium and Praseodymium Hydrides}

The original metals were: La $\alpha$ (hexagonal close packed arrangement, $a_{0}=3 \cdot 75_{7} \mathrm{~A}$., $\left.c / a=1 \cdot 61\right)$; Ce $\beta$ (face centred cubic, $a_{0}=5 \cdot 14 \mathrm{~A}$.; $\operatorname{Pr} \propto$ (hexagonal close packed, $a_{0}=3 \cdot 65_{2}, c / a=1 \cdot 61$ ).

Lanthanum annealed in vacuum at $350^{\circ} \mathrm{C}$., for several days, furnished powder photographs similar to those described very accurately by Zintl and Neumayr ${ }^{\lambda}$ for the $\beta$ phase (face centred cubic) of this element. I have noticed that by removing a very thin outer layer from the annealed specimens, the latter gave again the characteristic photographs of the $\alpha$ modification, that is, it was merely a surface phenomenon.

Praseodymium subjected to the same thermal treatment did not modify its structure. The specimens annealed in vacuum at $750^{\circ} \mathrm{C}$. for 48 hours furnish photographs of a somewhat different aspect, but yet not corresponding to a possible allotropical $\beta$ form.

The difficulty of hydrogen absorption increased in the order cerium, lanthanum, praseodymium.
In any event, the thermal treatment which was necessary to start the hydrogen absorption, repeated in vacuum on some specimens of the last two elements ( $\alpha$ modification), did not change their crystal structure.

The hydrides of the above mentioned metals all showed face-centred cubic lattices, with sizes larger than those pertaining to the real or possible $\beta$ phases of the original pure elements.

Lanthanum hydride furnished in one case photographs revealing the simultaneous presence of two face-centred cubic phases, having for side $a_{0}^{\prime}=5 \cdot 62 \mathrm{~A}$.; $\quad a_{0}^{\prime \prime}=5 \cdot 70 \mathrm{~A}$. (hydrogen absorbed, about $140 \mathrm{c.mm}$. per gm.). Generally, however, only one face-centred cubic phase appeared, having a side of $a_{0}=5 \cdot 62-5 \cdot 63 \mathrm{~A}$. (hydrogen absorbed, about $200 \mathrm{c} . \mathrm{mm}$. per gm.). If the hydrogen was removed by heating and a vacuum pump (at $1 \mathrm{~mm}$. pressure) the size of the lattice seemed to increase a little (at $530^{\circ} \quad a_{0}=\mathbf{5 . 6 5} \mathrm{A}$.; at $700^{\circ}$ slightly greater values).

Cerium hydride showed a lattice with side $a_{0}=5 \cdot 61_{2} \mathrm{~A}$. which by removal of the hydrogen in vacuum at $530^{\circ}$ split up into two similar phases of slightly smaller dimensions.

The lattice of praseodymium hydride (absorbed hydrogen, about $165 \mathrm{cmm}$.) was only slightly larger (some hundredths of an angström) than that of metallic cerium, that is, than that which a hypothetical $\beta$ phase of praseodymium would give, according to the small existing difference between the atomic diameters of praseodymium and cerium in the $\alpha$ phase.

\section{Istituto di Chimica generale e Chimica Fisica,}

Armando Rosst.

R. Università di Firenze.

${ }^{1}$ Z. Elekt, ang. phys. Chem., 39, Nr. 2, 84; 1933.

\section{Magnetic Anisotropy of Graphite}

GrapHITE is known from the investigations of Owen, Honda and others to exhibit an exceptionally large magnetic anisotropy. The susceptibilities of the natural crystal along its hexagonal axis and along perpendicular directions are, according to Honda :

$$
\chi_{1}=-14.2 \times 10^{-6} ; \chi_{2}=-2.2 \times 10^{-6}
$$

respectively, per gm., $\chi_{1}$ being thus more than six times $\chi_{2}$. Recently Goetz and his collaborators have found a much higher value for the ratio $\chi_{1} / \chi_{2}$. Chemically treated pure graphite powder is dispersed by them in a solution of gum Dammar in benzene; the solution is placed in a strong magnetic field and the benzene is allowed to evaporate. All the graphite particles in the solidified medium will then naturally be oriented in the same manner, namely, with their hexagonal axes normal to the direction of the imposed field. From susceptibility measurements on this medium they found ${ }^{2}$ for $\chi_{1} / \chi_{2}$ a value of $13 \cdot 2$. Later ${ }^{3}$, using graphite particles dispersed in this manner in a solidified solution of agar, they obtained a still higher value, namely, 18. Their more recent estimate ${ }^{4}$, obtained from a similar suspension of graphite particles in gelatine, is so high as 28 . It would thus seem desirable to determine the anisotropy of graphite by an independent method.

The following measurements made with some good specimens of Ceylon graphite, by Messrs. B. C. Guha and B. P. Roy in this laboratory, may therefore be of interest. The method adopted in these 